\title{
An Overview of Meta-Analyses of Endovascular Bridging Therapies for Acute Ischemic Stroke
}

\author{
Si-Yan Chen, ${ }^{1}$ Xing-Ru Zhang, ${ }^{1}$ Jie Chen, ${ }^{1}$ Wan-Qian Ge, ${ }^{1}$ Wen-Wen Wang, \\ Xin-Shi Wang $\left(\mathbb{1},{ }^{1} \text { and Cheng-Long Xie }{ }^{1}\right)^{1}$ \\ ${ }^{1}$ Department of Neurology, The First Affiliated Hospital of Wenzhou Medical University, Wenzhou 325000, China \\ ${ }^{2}$ The Center of Traditional Chinese Medicine, The Second Affiliated Hospital and Yuying Children's Hospital of \\ Wenzhou Medical University, Wenzhou 325027, China
}

Correspondence should be addressed to Xin-Shi Wang; 33899486@qq.com and Cheng-Long Xie; cl_xie1987@sohu.com

Received 22 July 2017; Revised 16 November 2017; Accepted 18 December 2017; Published 7 March 2018

Academic Editor: Massimo Collino

Copyright (C) 2018 Si-Yan Chen et al. This is an open access article distributed under the Creative Commons Attribution License, which permits unrestricted use, distribution, and reproduction in any medium, provided the original work is properly cited.

\begin{abstract}
Background. Acute Ischemic Stroke (AIS) is a common cause of death worldwide and the leading cause of long-term severe disability. Endovascular bridging therapies (EBT), including endovascular thrombectomy (ET) and intra-arterial thrombolytic (IAT), have been recommended to realize a favorable functional outcome for AIS patients. Methods. An overview of meta-analyses of primary randomized controlled trial (RCT) studies was performed evaluating EBT for AIS patients compared with usual care. Results. Ten meta-analyses were included in this overview. ET was associated with a higher incidence of achieving functional outcome improvement, defined as a modified Rankin scale of 0 to 1 (mRS, $p=0.003), 0$ to $2(p<0.00001)$, and 0 to $3(p=0.005)$. The risk of symptomatic intracranial hemorrhage (sICH) rate and all-cause mortality were similar between the two groups. Moreover, IAT treatment was also related to significantly improved outcomes in terms of the mRS score $(p<0.05)$, but no significant difference in rates of sICH and mortality within 90 days. Conclusions. In conclusion, our analysis supports that EBT, regardless of format (e.g., ET or IAT), is superior to the best medical therapy alone (e.g., IVT) in terms of mRS score in patients with AIS. In addition, the safety of EBT is similar to IVT.
\end{abstract}

\section{Introduction}

Stroke is a common cause of death worldwide and the leading cause of long-term severe disability. Over $80 \%$ of all incident strokes are ischemic, resulting from an occluding thrombus of a cerebral artery [1]. Intravenous recombinant tissue plasminogen activator (rt-PA) administered as early as possible within 4.5 hours is recommended after the onset of acute ischemic stroke (AIS) which improves survival rate and functional outcomes [2] and is the most proven helpful therapy for the emergency management of AIS [3]. Nevertheless, intravenous rt-PA (IVT) has multiple limitations, containing nonresponsiveness of large thrombus to rapid dissolution, a narrow time window for treatment [4], the risk of cerebral and various systems hemorrhage, and several important contraindications, such as recent surgery, coagulation abnormalities, or head injury within the past 3 months [5]. Consequently, as few as $10 \%$ of AIS patients can be qualified for administration with IVT [6]. Furthermore, some AIS patients with intracranial obstruction of internal carotid artery (ICA) or the middle cerebral artery (MCA) are associated with long reperfusion times and poor revascularization rates, IVT results in early recanalization approximate 10 to $50 \%$ [7], and the prognosis of these patients remains poor, which lead to exploration of new endovascular bridging therapy for AIS patients [8].

Endovascular bridging therapies (EBT) include intraarterial pharmacologic thrombolysis (IAT) or manipulation of the clot with the use of stent-retriever technology after IVT. EBT are given through the large arteries more frequently and rapidly than IVT in patients with AIS and are utilized to cure the patients with occlusions of the large intracranial arteries [9]. Endovascular treatment of AIS with IAT has been revealed as a vital therapeutic modality [10] and conferred a markedly greater chance of realizing favorable functional outcomes compared with IVT for AIS patients [11]. Meanwhile, 
endovascular thrombectomy (ET) with mechanical devices is a straight reperfusion way that is different from pharmacologic thrombolysis [12]. Endovascular mechanical treatments can recanalize large arterial occlusions and remove proximal clots rapidly and lead to higher rates of reperfusion than IVT alone [13]. At this stage, it is significant to establish the efficacy and safety of EBT in patients with AIS and furthermore to address the pivotal factors that determine their success or failure. Until now, several systematic reviews or metaanalyses have been conducted to assess EBT in treatment of AIS. We will comment on this evidence in light of the overall effectiveness of EBT interventions in the separate systematic reviews. By doing so, to bring together all relevant published information based on the large amount of available data, we here offer an overview of those published systematic reviews.

\section{Materials and Methods}

We implemented this overview of meta-analyses based on the modified Preferred Reporting Items for Systematic Reviews and Meta-Analyses (PRISMA) statement (see the additional file: PRISMA checklist (available here)).

2.1. Search Strategy. Systematic literature searches of PubMed, Cochrane Library, and Google Scholar databases published from their inception to January 2017 were performed to identify studies addressing the role of EBT (containing intra-arterial thrombolysis and endovascular thrombectomy after intravenous rt-PA) in AIS patients management. The search terms used were various relevant combinations: "endovascular or intra-arterial or fibrinolysis or thrombolysis or thrombectomy" AND "ischemia or stroke" AND "systematic review or meta-analysis". Reference lists of studies with potential all relevant articles were manually screened for additional studies that were not previously identified.

2.2. Inclusion and Exclusion Criteria. For this overview of reviews, we included only systematic reviews or metaanalyses of RCTs (randomized controlled trials) of EBT in participants with AIS. The included systematic reviews had to live up to the following criteria.

Participants. Adults (aged 18 years and over) who reported to suffer acute ischemic stroke within 12 hours were included.

Interventions and Comparison. Meta-analyses of RCTs assessing "intra-arterial administration of thrombolytic drugs (e.g., IAT) or the use of various thrombectomy devices (e.g., ET)" after IVT as the intervention compared with IVT alone were considered. IAT was known as chemical dissolution of blood clots with locally delivered thrombolytic; ET was defined as the intra-arterial utilization of a micropipe or other devices for mechanical thrombectomy.

Primary Outcomes and Secondary Outcomes. The primary outcome measure is the functional index using the modified Rankin scale (mRS). This 7-score ordinal scale ranges from
0 (no symptoms) to 6 (death). Secondary outcomes studied were symptomatic intracranial hemorrhage (sICH), failure to recanalize, and mortality.

2.3. Data Extraction and Analysis. All systematic reviews were read by two independent authors (WQG, JC) and data from the studies were extracted using a Microsoft Excel spreadsheet and validated according to predefined criteria. Original, two authors (WQG, JC) received a detailed introduction to the overview protocol and then independently screened a small sample of records to pilot test screening forms. In case data from the systematic reviews were unclear or seemed to be missing, we touched the correspondence authors for statement and found original RCTs for details. Any disagreements or disputes were resolved through discussion, and a third overview author (CLX) acted as a referee if necessary. We extracted and summarized the following details from the included article: author's name, the publication year, number of included studies and participants, type of intervention, comparator, average time from onset to treatment, length of follow-up, outcome measures, and the conclusions of the included meta-analyses.

2.4. Quality Assessment of Included Reviews. Eligible systematic reviews were quality assessed using AMSTAR questionnaire, an instrument used to evaluate the methodological quality of systematic reviews [14] and the degree to which reviews are biased by comparing them on the basis of distinct criteria. Eleven AMSTAR items are as follows: (1) Was a priori design provided? (2) Was a comprehensive and detailed literature search performed? (3) Was there duplicate study selection and data extraction? (4) Was the status of publication used as an inclusion criterion? (5) Was a list of included and excluded studies provided? (6) Were the basic characteristics of included studies provided? (7) Was the scientific quality of the included studies assessed and documented? (8) Was the scientific quality of the included studies used appropriately in formulating conclusions? (9) Were the methods used to combine the findings of studies appropriate? (10) Was the likelihood of publication bias assessed? (11) Was the conflict of interest stated? Ratings used in AMSTAR include "yes" (clearly done), "no" (clearly not done), and "not applicable."

\section{Results}

3.1. Description of the Screening Process. The searches identified potential meta-analyses and the total number of papers found in three database was 361. After eliminating duplicate papers, 209 papers remained. Through screening the titles and abstracts, we further ruled out 111 papers in this stage. In the remaining 98 papers, after checking the full texts, finally 10 meta-analyses were included in this overview [15-24]. Among them, seven meta-analyses focused on the use of ET after IVT as the intervention compared with IVT alone, whilst two studies compared IAT + IVT with IVT, and the remaining one study compared EBT with IVT. The detail of search process is shown in Figure 1. 


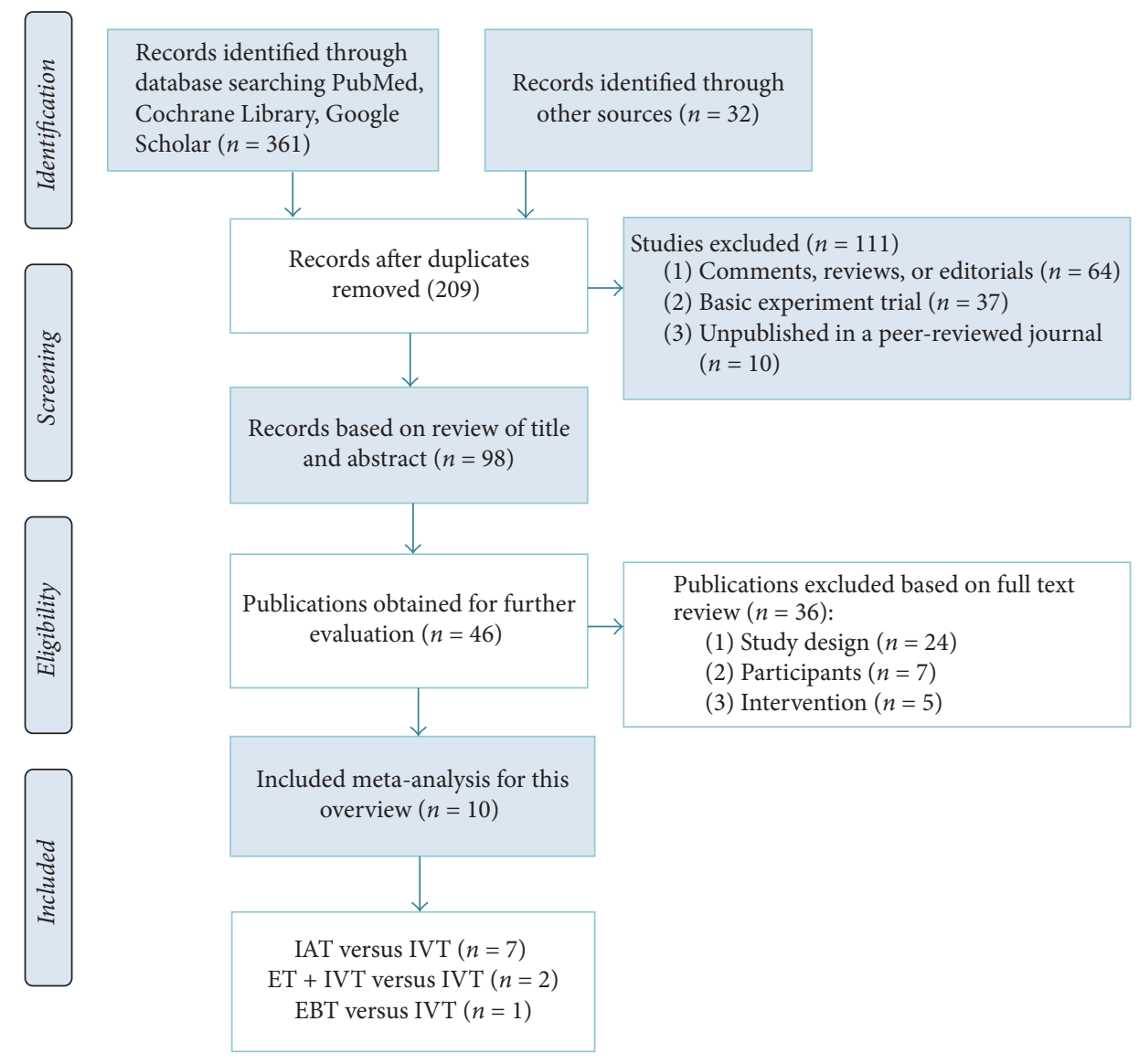

FIgURE 1: Study flow diagram.

3.2. Study Characteristics and Assessing the Quality of MetaAnalysis. We selected the most recent updates of metaanalyses of thrombectomy devices (ET) or intra-arterial administration of thrombolytic drugs (IAT) for treating AIS compared with IVT for inclusion in this overview. Relevant primary studies in the included 10 meta-analyses were demonstrated in Table 1 . We found that meta-analyses included between 3 and 9 primary studies meeting the inclusion criteria and some studies were included in multiple meta-analyses, especially five studies of MR CLEAN, ESCAPE, EXTEND-IA, SWIFT PRIME, and REVASCAT. The basic characteristics and major conclusions of included meta-analyses are shown in Table 2 . The 10 meta-analyses included were published between 2010 and 2016. Following quality assessment using AMSTAR, nearly all eligible metaanalyses were deemed high quality and included for full review. All meta-analyses reported mRS score at 3 months as a primary outcome and nine studies reported symptomatic intracranial hemorrhage and mortality as a secondary outcome. Nevertheless, only two studies showed the data about revascularization at 24 hours. In this overview, no metaanalysis achieved a perfect score of 11/11, though six achieved $10 / 11$, three scored 9/11, and the remaining one got 8/11. Quality assessment results are displayed in Table 2. Criteria which scored badly using the AMSTAR tool were "provide a priori design (question 1)" and "the status of publication used as an inclusion criterion (question 4).”

3.3. ET + IVT versus IVT. Pooling the result of mRS score was more favorable with ET relative to standard therapy, with greater proportions of AIS patients in each category of favorable outcome (mRS $0-1,0-2$, or $0-3$, resp.). For the primary outcome of mRS score reduction, four studies [18-21] consistently showed reduced chance of disability at 3 months in patients assigned to ET versus those assigned to control $(p<$ 0.05 , Table 3$)$. In terms of $\mathrm{mRS}$ score (0-1), ET was associated with significantly higher rates of functional independence at 3 months (OR 1.89; 95\% CI: 1.25-2.85; $p=0.003$; Figure 2); mRS score (0-2) was available from 6 trials of seven; then we pooled the whole data and found significant difference in favor of ET compared to IVT (OR 1.73; 95\% CI: 1.39-2.15; $p<0.00001$; Figure 2); meanwhile, a statistical significant effect was obtained between ET and IVT based on the mRS score $0-3$ (OR 1.46; 95\% CI: 1.12-1.90; $p=0.005$; Figure 2).

Secondary outcomes of mortality and sICH rate at 3 months among both groups for all trials are detailed in Table 4 . There were six meta-analyses that mentioned mortality and sICH rate and the results were not difference at all. Singh et al. [15] showed that ET is not superior to IVT in improving mortality (17.9\% versus $17.1 \%, p>0.05)$ with 


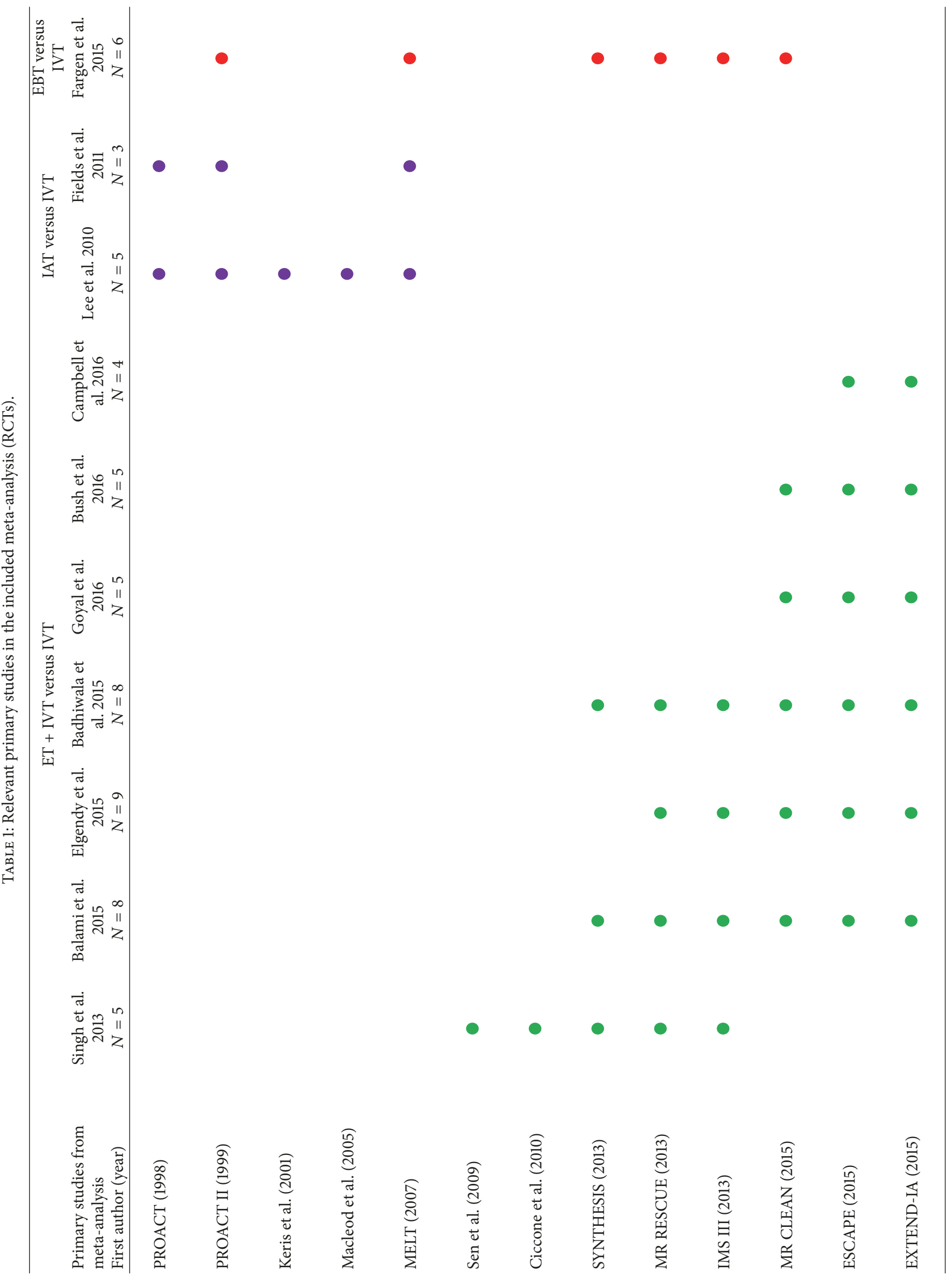




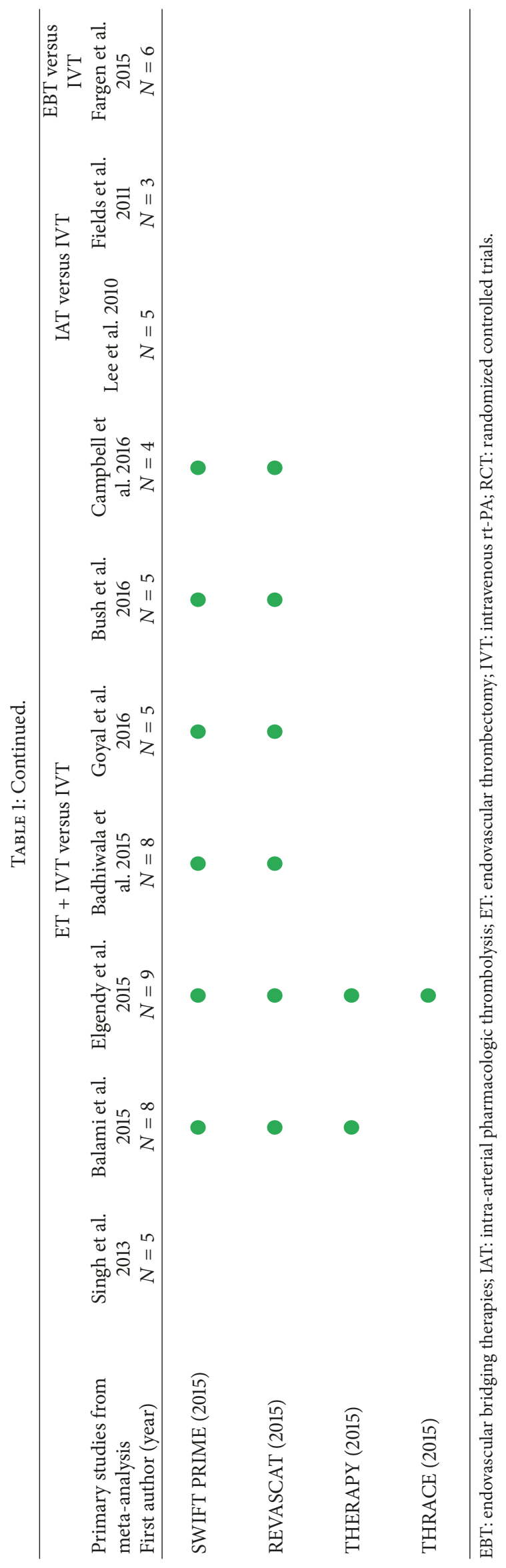




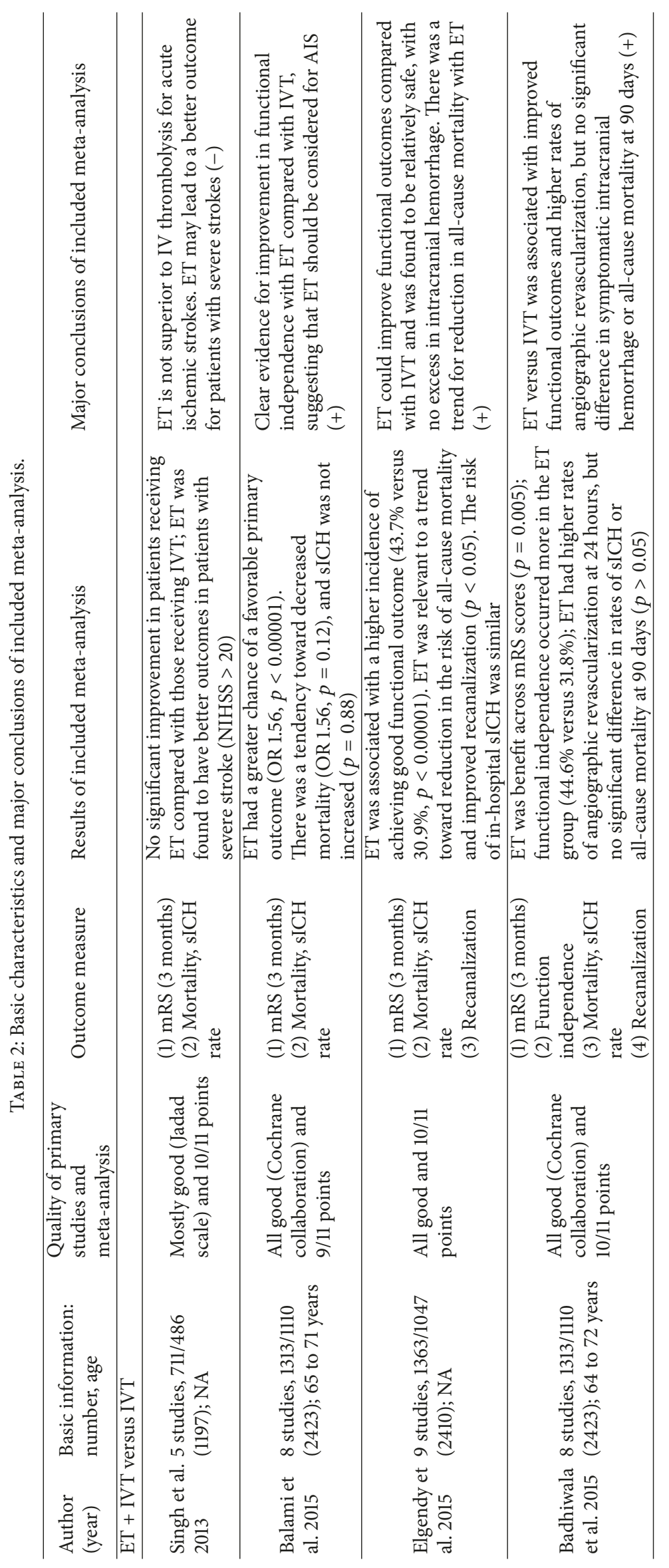




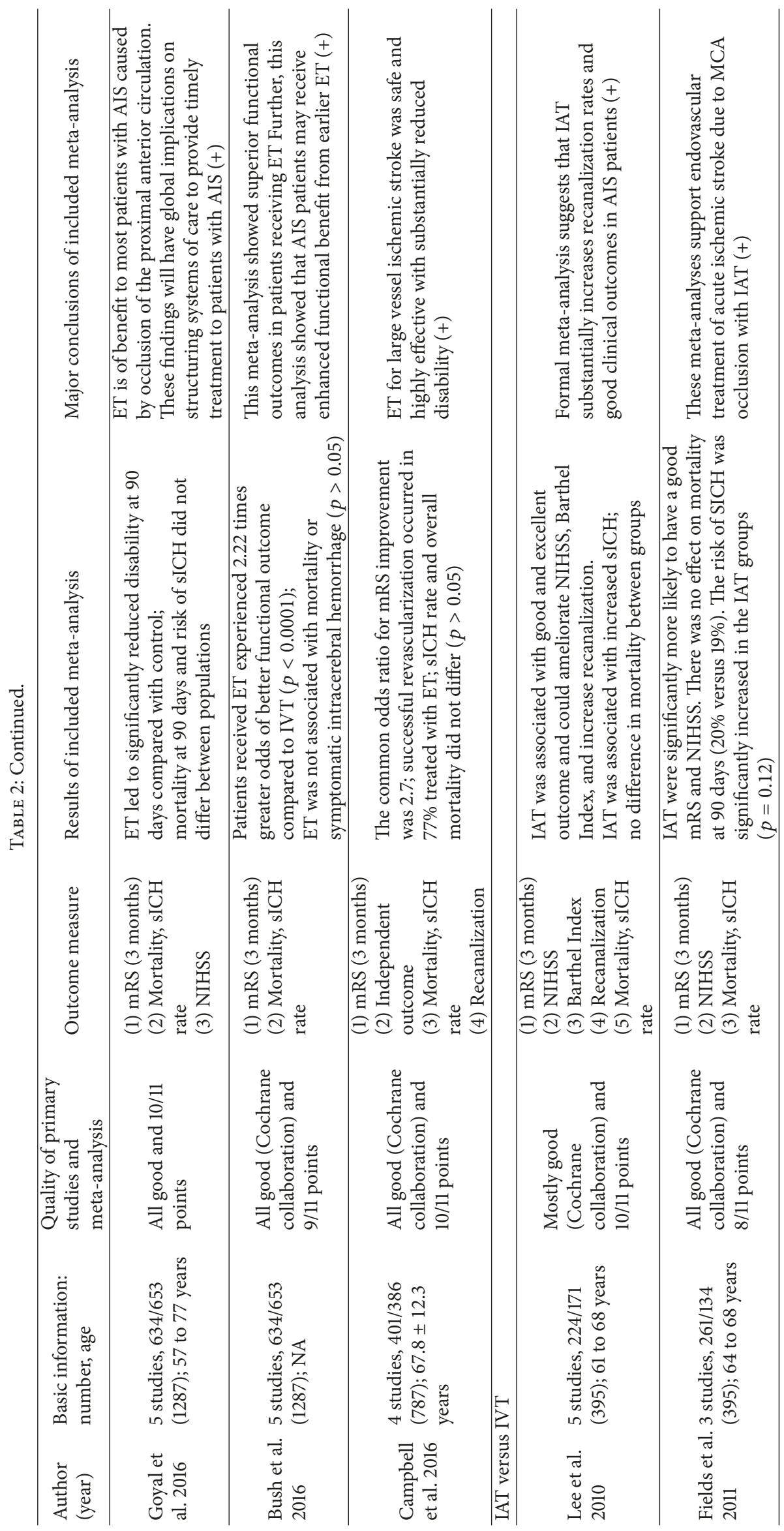




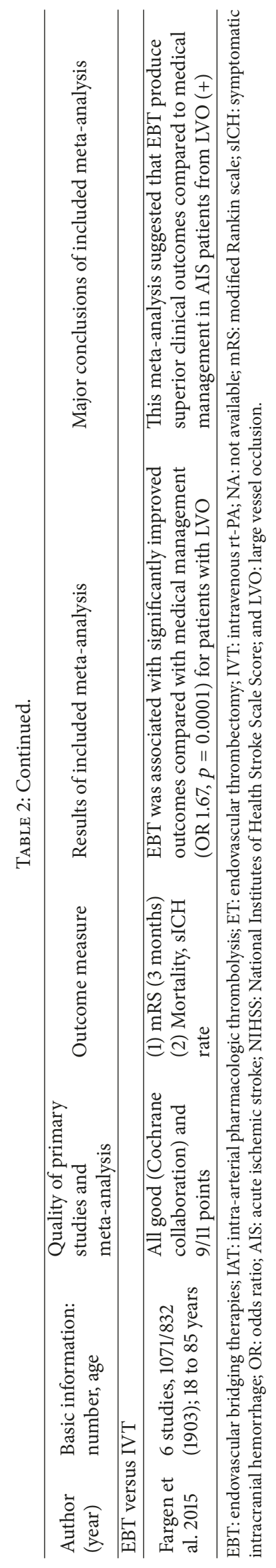


TABLE 3: Efficacy outcomes of mRS score from the pooled data.

\begin{tabular}{|c|c|c|c|}
\hline Author (year) & OR & $95 \%$ CI & $p$ value \\
\hline \multicolumn{4}{|c|}{ ET + IVT versus IVT: mRS score (0-6) } \\
\hline Badhiwala et al. 2015 & 1.56 & $1.14-2.13$ & 0.0005 \\
\hline Goyal et al. 2016 & 2.26 & $1.67-3.06$ & 0.0001 \\
\hline Bush et al. 2016 & 2.47 & $1.92-3.18$ & 0.0001 \\
\hline Campbell et al. 2016 & 2.4 & $1.8-3.0$ & 0.0001 \\
\hline \multicolumn{4}{|c|}{ IAT versus IVT: mRS score $(0-1)$} \\
\hline Lee et al. 2010 & 2.14 & $1.31-3.51$ & 0.003 \\
\hline Fields et al. 2011 & 1.97 & $1.15-3.35$ & 0.01 \\
\hline \multicolumn{4}{|c|}{ IAT versus IVT: mRS score $(0-2)$} \\
\hline Lee et al. 2010 & 2.05 & $1.33-3.14$ & 0.001 \\
\hline Fields et al. 2011 & 1.86 & $1.15-2.99$ & 0.01 \\
\hline \multicolumn{4}{|c|}{ EBT versus IVT: Fargen et al. 2015} \\
\hline mRS score $(0-1)$ & 1.22 & $0.97-1.53$ & 0.09 \\
\hline mRS score $(0-2)$ & 1.27 & $1.04-1.54$ & 0.018 \\
\hline mRS score $(0-3)$ & 1.25 & $1.04-1.51$ & 0.019 \\
\hline
\end{tabular}

EBT: endovascular bridging therapies; ET: endovascular thrombectomy; IAT: intra-arterial pharmacologic thrombolysis; IVT: intravenous rt-PA; mRS: modified Rankin scale; OR: odds ratio; and 95\% CI: 95\% confidence interval.

a similar rate of symptomatic hemorrhage (5.9\% versus $6.1 \%$, $p>0.05$ ). One study conducted by Balami et al. [16] demonstrated that no significant difference was found in the prespecified secondary outcomes mortality (OR 0.84; 95\% CI: $0.64-1.05 ; p=0.12$ ) and sICH (OR 1.03; 95\% CI: 0.71-1.49; $p=0.88)$ when ET was compared with IVT. However, Elgendy et al. [17] displayed that ET after usual care was associated with a trend toward reduction in the risk of allcause mortality $(15.9 \%$ versus $17.9 \%, p=0.82)$ and sICH rate (5.1\% versus $5.0 \%, p=1.02)$ compared with usual care alone, but still not significant. Similarly, mortality at 90 days and risk of sICH rate did not differ between populations in the remaining three meta-analysis (mortality: $p=0.27, p=0.16$, and $p=0.12$, resp.; sICH: $p=0.56, p=0.8$, and $p=0.76$, resp.). In addition, the results of revascularization at 24 hours were conclusive in the two meta-analyses. Elgendy et al. [17] reported that ET after usual care was related to improved recanalization compared with usual care alone (66.6\% versus $39.2 \%, p=0.0001)$. Meanwhile, one meta by Badhiwala et al. [18] showed that rates of angiographic revascularization at 24 hours for ET were $75.8 \%$ versus $34.1 \%$ for standard therapy (OR 6.49; 95\% CI: 4.79-8.79; $p<0.01$ ). Efficacy of secondary outcomes from the pooled data is shown in Table 4.

3.4. IAT + IVT versus IVT. We identified two meta-analyses comparing IAT with IVT in AIS patients. Pooling the results conducted by Lee et al. [22] reported that IAT was associated with increased good clinical outcomes according to $\mathrm{mRS}$ score 0-2 (OR 2.05; 95\% CI: $1.33-3.14 ; p=0.001$ ) and excellent clinical outcomes based on the mRS score $0-1$ (OR 2.14; 95\% CI: $1.31-3.51 ; p=0.003)$. For additional end points, IAT also increased frequencies of minimal neurologic deficit and reduced impairment of activities of daily living and partial or complete recanalization. Fields et al. [23] indicated that IAT treated patients were significantly more likely to have a mRS $0-1$ (31\% versus $20 \%, p=0.01)$; mRS $0-2(43 \%$ versus $31 \%, p=0.01)$; and NIHSS score $(p=$ 0.007 ) at 3 -month follow-up. Table 3 showed the detailed data of the included meta-analyses. In terms of safety question, both meta-analyses reported that IAT was associated with increased $\mathrm{sICH}$ rate $(8.9 \%$ versus $2.3 \%, p=0.02 ; 11 \%$ versus $2 \%, p=0.02$, resp.). However, there was no difference in mortality between IAT and IVT groups (20.5\% versus $24.0 \%$, $p=0.46 ; 20.0 \%$ versus $19.0 \%, p=0.57$, resp.). Consequently, increased $\mathrm{sICH}$ frequencies are not associated with any increase in mortality based on these two meta-analyses.

3.5. EBT versus IVT. Only one meta-analysis comparing EBT with IVT in AIS patients indicated that the general effectiveness of EBT was much better than IVT. Fargen et al. [24] reported that primary outcome (mRS 0-2 at 3 months) occurred significantly more frequent in patients randomized to EBT compared with IVT (39.1\% versus $32.6 \%, p=0.018$, Table 3). Conversely, the secondary outcome of mRS $0-1$ did not show significant discrepancy between two groups $(p=$ 0.09 ). Finally, mRS 0-3 at 90 days occurred markedly more continually in the EBT $\operatorname{arm}(p=0.019)$ and the mortality did not show obvious difference between the two groups $(p=$ $0.73)$.

\section{Discussion}

4.1. Summary of Evidence. Ten meta-analyses were included in the present overview. To our knowledge, this is the first overview for meta-analyses on efficacy and safety of EBT for AIS patients. We found that EBT, regardless of format (e.g., ET or IAT), is superior to the best medical therapy alone (e.g., IVT) in terms of mRS score in patients with acute ischemic stroke. Compared with control groups, adverse events were similar in patients treated with EBT compared with IVT regarding the symptoms of intracranial hemorrhage and mortality risk did not apparently differ between groups.

4.2. Thrombectomy. The conclusions that can be drawn about the efficacy of thrombectomy for AIS are solid because they are based on seven meta-analyses and several large multicenter case registries. One special advantage of our meta-analysis is that it contains only trials that incorporated crucial elements of current clinical practice, such as mechanical thrombectomy after usual care (e.g., intravenous alteplase) and universal requirement for proven large artery occlusion and timely treatment (within $6 \mathrm{~h}$ ). Meanwhile, three meta-analyses [19-21] utilized the second-generation stent retrievers and demonstrated robust benefit of such mechanical device for AIS patients, approaching a double increase in odds of achieving a beneficial shift in mRS score. Goyal et al. [19] and Campbell et al. [21] reported that the extent of benefit conferred by ET is substantial; for every 100 patients treated, approximately 40 will have a less disabled outcome than with IVT, and nearly 23 more will achieve an independent outcome (mRS $0-2$ ) as a result of treatment. Overall, all meta-analyses included in this overview showed 


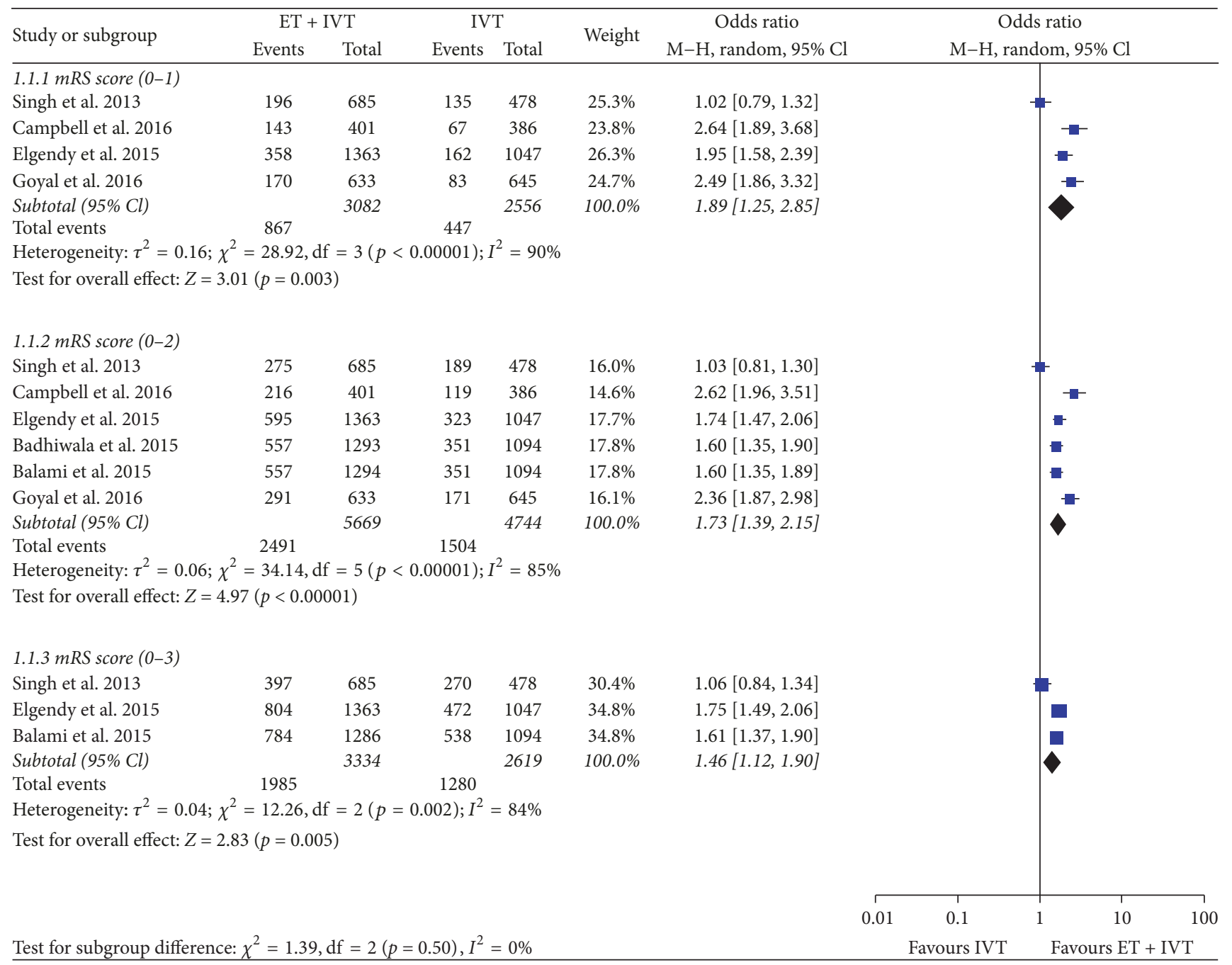

FIGURE 2: Meta-analysis of EBT versus standard therapy for the outcome of proportional treatment benefit across mRS scores (0 to 1, 0 to 2 , and 0 to 3, resp.) at 90 days. EBT: endovascular bridging therapies; mRS: modified Rankin scale.

a trend for better outcomes with ET. Moreover, advanced and detailed imaging before ET leads to a delay in the time to revascularization. Elgendy et al. [17] demonstrated that advanced imaging has not been shown to affect patient outcomes or increase the risk of sICH rate, but it helps to select appropriate patients for ET.

4.3. Intra-Arterial Thrombolysis. Only two meta-analyses [22, 23] mentioned IAT treatment after IVT as the intervention compared with IVT alone for AIS patients and the results were significant. In one meta-analysis [23], the data pooled from three studies provide a precise estimate of the profitable effect of IAT when administered within 6 hours of symptom occurrence for the therapy of AIS due to middle cerebral artery (MCA) occlusion. Although a total of just 334 AIS patients were enrolled in these three studies and the number of studies and patients was limited, a clinically and statistically robust increase in the probability of a favorable functional outcome mainly according to mRS score was observed in the IAT compared with control subjects. Three primary studies included in this meta-analysis have similar time window $(<6 \mathrm{~h})$ and artery occluded (M1 segment of the MCA); all of these factors contribute to the homogeneous results and are easy to generalize. Moreover, although this overall benefit came at the cost of increased risk of sICH rate, with an OR of 4.6 in the IAT treated group, there were no influence and discrepancy on 90-day mortality. Another meta-analysis conducted by Lee et al. 2010 [22] based on five studies showed that beneficial effects of IAT after IVT were highly statistically difference for increased rates of both good $(14.8 \%)$ and excellent $(13.0 \%)$ clinical outcomes. The chances of these outcome measures were more than doubled compared to control groups.

4.4. Adverse Effects. All the treatments considered in this overview carry the risk of side-effects (mortality and sICH rate). There were nine meta-analyses [15-19, 21-24] that mentioned mortality and the results were not difference at all (Table 4). In terms of sICH rate, the risk of in-hospital sICH was similar between ET and IVT groups based on six 
TABLE 4: Efficacy of secondary outcomes from the pooled data.

\begin{tabular}{|c|c|c|c|c|c|c|c|c|c|}
\hline \multirow[b]{2}{*}{$\begin{array}{l}\text { Primary studies of } \\
\text { meta-analysis }\end{array}$} & \multirow[b]{2}{*}{$\begin{array}{l}\text { Singh et al. } \\
2013\end{array}$} & \multirow[b]{2}{*}{$\begin{array}{l}\text { Balami et } \\
\text { al. } 2015\end{array}$} & \multicolumn{2}{|c|}{$\mathrm{ET}+\mathrm{IVT}$ versus IVT } & \multirow[b]{2}{*}{$\begin{array}{l}\text { Goyal et } \\
\text { al. } 2016\end{array}$} & \multirow[b]{2}{*}{$\begin{array}{l}\text { Campbell } \\
\text { et al. } 2016\end{array}$} & \multicolumn{2}{|c|}{ IAT versus IVT } & \multirow{2}{*}{$\begin{array}{l}\text { EBT versus } \\
\text { IVT } \\
\text { Fargen et al. } \\
\quad 2015\end{array}$} \\
\hline & & & $\begin{array}{c}\text { Elgendy et al. } \\
2015\end{array}$ & $\begin{array}{c}\text { Badhiwala et } \\
\text { al. } 2015\end{array}$ & & & $\begin{array}{c}\text { Lee et al. } \\
2010\end{array}$ & $\begin{array}{c}\text { Fields et al. } \\
2011\end{array}$ & \\
\hline \multicolumn{10}{|l|}{ Mortality } \\
\hline $\mathrm{N} 1 / \mathrm{T} 1$ & $127 / 707$ & $206 / 1312$ & $15.9 \%$ & $218 / 1312$ & $97 / 633$ & $48 / 401$ & $46 / 224$ & $40 / 201$ & $203 / 1071$ \\
\hline $\mathrm{N} 2 / \mathrm{T} 2$ & $84 / 490$ & $194 / 1106$ & $17.9 \%$ & $201 / 1106$ & $122 / 646$ & $63 / 386$ & $41 / 171$ & $24 / 130$ & $156 / 832$ \\
\hline $\begin{array}{l}\text { OR } \\
95 \% \mathrm{CI}\end{array}$ & $\begin{array}{c}0.98 \\
(0.76-1.25)\end{array}$ & $\begin{array}{c}0.84 \\
(0.64-1.05)\end{array}$ & $\begin{array}{c}0.82 \\
(0.67-1.02)\end{array}$ & $\begin{array}{c}0.87 \\
(0.68-1.12)\end{array}$ & $\begin{array}{c}0.77 \\
(0.54-1.10)\end{array}$ & $\begin{array}{c}0.69 \\
(0.43-1.1)\end{array}$ & $\begin{array}{c}0.83 \\
(0.48-1.39)\end{array}$ & $\begin{array}{c}0.84 \\
(0.47-1.52)\end{array}$ & $\begin{array}{c}0.96 \\
(0.76-1.22)\end{array}$ \\
\hline$p$ value & $>0.05$ & 0.12 & 0.08 & 0.27 & 0.16 & 0.12 & 0.46 & 0.57 & 0.73 \\
\hline \multicolumn{10}{|l|}{ sICH rate } \\
\hline N1/T1 & $42 / 707$ & $66 / 1312$ & $5.1 \%$ & $70 / 1312$ & $28 / 633$ & $10 / 401$ & $20 / 224$ & $20 / 201$ & \\
\hline $\mathrm{N} 2 / \mathrm{T} 2$ & $30 / 490$ & $53 / 1106$ & $5.0 \%$ & $53 / 1106$ & $28 / 646$ & $11 / 386$ & $4 / 171$ & $3 / 130$ & \\
\hline $\begin{array}{l}\text { OR } \\
95 \% \mathrm{CI}\end{array}$ & $\begin{array}{c}0.99 \\
(0.62-1.58)\end{array}$ & $\begin{array}{c}1.03 \\
(0.71-1.49)\end{array}$ & $\begin{array}{c}1.02 \\
(0.69-1.52)\end{array}$ & $\begin{array}{c}1.12 \\
(0.77-1.63)\end{array}$ & $\begin{array}{c}1.07 \\
(0.62-1.83)\end{array}$ & $\begin{array}{c}0.87 \\
(0.36-2.1)\end{array}$ & $\begin{array}{c}2.87 \\
(1.21-6.83)\end{array}$ & $\begin{array}{c}4.58 \\
(1.31-15.97)\end{array}$ & \\
\hline$p$ value & $>0.05$ & 0.88 & 0.92 & 0.56 & 0.8 & 0.76 & 0.02 & 0.02 & \\
\hline \multicolumn{10}{|l|}{ Revascularization } \\
\hline $\begin{array}{l}\text { OR } \\
95 \% \mathrm{CI}\end{array}$ & & & $\begin{array}{c}3.09 \\
(2.46-3.89)\end{array}$ & $\begin{array}{c}6.49 \\
(4.79-8.79)\end{array}$ & & & & & \\
\hline$p$ value & & & 0.0001 & $<0.01$ & & & & & \\
\hline
\end{tabular}

EBT: endovascular bridging therapies; ET: endovascular thrombectomy; IAT: intra-arterial pharmacologic thrombolysis; IVT: intravenous rt-PA; mRS: modified Rankin scale; OR: odds ratio; and 95\% CI: 95\% confidence interval.

meta-analyses. Nonetheless, two meta-analyses [22, 23] of IAT compared with IVT showed increased sICH frequencies; however, the results are not associated with any increase in mortality. The sample size of included studies in this review was enough for quantification of serious adverse events and found no difference between two arms. In a word, the safety of EBT is similar to IVT in clinic. The results of such metaanalyses could show the development of clinical practice guidelines. In addition, it may be beneficial for medical personnel involved in the early care of patients with AIS and using ET (after IVT) as the first choice when encountering candidate patients compared with IVT alone.

4.5. Limitations. There are some limitations to this overview. First, the included meta-analyses were heterogeneous and were not necessarily comparable. They were carried out on different regions, with different enrollment criteria, and used variable endovascular therapies tools. Second, all of the included meta-analyses specified that AIS patients were included on the basis of CT imaging, and EBT was conducted quickly once imaging eligibility had been ascertained, whereas the benefit of EBT was not analysis alone for some of patients with poor collateral grade or unfavorable penumbral patterns in this paper. The capacity to provide adjusted treatment effect for some subgroups analysis was limited. Third, three included meta-analyses [19-21] focused on the endovascular trials using the Solitaire device and the remaining four utilized other endovascular devices or Solitaire mix. The direct comparison was missing and unable to draw the definite conclusions which was better. However, several studies confirmed the robust treatment benefits when using the Solitaire device in AIS patients with large vessel occlusion ischemic stroke.

\section{Conclusions}

In conclusion, our analysis supports that EBT, regardless of format (e.g., ET or IAT), is superior to the best medical therapy alone (e.g., IVT) in terms of mRS score in patients with AIS. In addition, the safety of EBT is similar to IVT.

\section{Conflicts of Interest}

The authors declare that they have no conflicts of interest.

\section{Authors' Contributions}

Cheng-Long Xie and Si-Yan Chen conceived and participated in the study design and searched the databases. Xing-Ru Zhang and Wan-Qian Ge extracted and assessed studies and helped to draft the manuscript. Jie Chen and Xin-Shi Wang helped in guiding and revising the manuscript. WenWen Wang participated in the conceptualization and design of the review and revised the review. All authors read and approved the final manuscript. Si-Yan Chen and Xing-Ru Zhang contributed equally to this work.

\section{Acknowledgments}

The authors gratefully acknowledge Dr. Man-Man Zhang from Wenzhou Medical University for his help in guiding and revising the manuscript. The study was supported by the 
projects of the National Science Foundation of China (no. 81600977).

\section{Supplementary Materials}

2009 PRISMA Checklist for meta-analysis. (Supplementary Materials)

\section{References}

[1] D. Lloyd-Jones, R. J. Adams, T. M. Brown et al., "Heart disease and stroke statistics-2010 update: a report from the American Heart Association," Circulation, vol. 121, no. 7, pp. e46-e215, 2010.

[2] W. Hacke, M. Kaste, E. Bluhmki et al., "Thrombolysis with alteplase 3 to 4.5 hours after acute ischemic stroke," The New England Journal of Medicine, vol. 359, no. 13, pp. 1317-1329, 2008.

[3] H. P. Adams Jr., G. Del Zoppo, M. J. Alberts et al., “The American Academy of Neurology affirms the value of this guideline as an educational tool for neurologists," Stroke, vol. 38, no. 5, pp. 1655-1711, 2007.

[4] K. R. Lees, E. Bluhmki, R. von Kummer et al., "Time to treatment with intravenous alteplase and outcome in stroke: an updated pooled analysis of ECASS, ATLANTIS, NINDS, and EPITHET trials," The Lancet, vol. 375, no. 9727, pp. 1695-1703, 2010.

[5] E. C. Jauch, J. L. Saver, H. P. Adams et al., "Guidelines for the early management of patients with acute ischemic stroke: a guideline for healthcare professionals from the American Heart Association/American Stroke Association," Stroke, vol. 44, no. 3, pp. 870-947, 2013.

[6] F. De Los Ríos La Rosa, J. Khoury, B. M. Kissela et al., "Eligibility for intravenous recombinant tissue-type plasminogen activator within a population: the effect of the European Cooperative Acute Stroke Study (ECASS) III trial," Stroke, vol. 43, no. 6, pp. 1591-1595, 2012.

[7] M. Paciaroni, C. Balucani, G. Agnelli et al., "Systemic thrombolysis in patients with acute ischemic stroke and Internal Carotid ARtery Occlusion: the ICARO study," Stroke, vol. 43, no. 1, pp. 125-130, 2012.

[8] M. Saqqur, K. Uchino, A. M. Demchuk et al., "Site of arterial occlusion identified by transcranial Doppler predicts the response to intravenous thrombolysis for stroke," Stroke, vol. 38, no. 3, pp. 948-954, 2007.

[9] P. M. Meyers, H. C. Schumacher, E. S. Connolly Jr., E. J. Heyer, W. A. Gray, and R. T. Higashida, "Current status of endovascular stroke treatment," Circulation, vol. 123, no. 22, pp. 2591-2601, 2011.

[10] A. I. Qureshi, A. M. Siddiqui, S. H. Kim et al., "Reocclusion of recanalized arteries during intra-arterial thrombolysis for acute ischemic stroke," American Journal of Neuroradiology, vol. 25, no. 2, pp. 322-328, 2004.

[11] Q.-F. Ma, C.-B. Chu, and H.-Q. Song, "Intravenous versus intraarterial thrombolysis in ischemic stroke: a systematic review and meta-analysis," PLoS ONE, vol. 10, no. 1, Article ID e0116120, 2015.

[12] B. C. V. Campbell, G. A. Donnan, K. R. Lees et al., "Endovascular stent thrombectomy: the new standard of care for large vessel ischaemic stroke," The Lancet Neurology, vol. 14, no. 8, pp. 846854, 2015.
[13] J. L. Saver, M. Goyal, A. Bonafe et al., "Stent-retriever thrombectomy after intravenous t-PA vs. t-PA alone in stroke," The New England Journal of Medicine, vol. 372, no. 24, pp. 2285-2295, 2015.

[14] B. J. Shea, J. M. Grimshaw, and G. A. Wells, "Development of AMSTAR: a measurement tool to assess the methodological quality of systematic reviews," BMC Medical Research Methodology, vol. 7, p. 10, 2007.

[15] B. Singh, A. K. Parsaik, L. J. Prokop, and M. K. Mittal, "Endovascular therapy for acute ischemic stroke: a systematic review and meta-analysis," Mayo Clinic Proceedings, vol. 88, no. 10, pp. 1056-1065, 2013.

[16] J. S. Balami, B. A. Sutherland, L. D. Edmunds et al., "A systematic review and meta-analysis of randomized controlled trials of endovascular thrombectomy compared with best medical treatment for acute ischemic stroke," International Journal of Stroke, vol. 10, no. 8, pp. 1168-1178, 2015.

[17] I. Y. Elgendy, D. J. Kumbhani, A. Mahmoud, D. L. Bhatt, and A. A. Bavry, "Mechanical thrombectomy for acute ischemic stroke a meta-analysis of randomized trials," Journal of the American College of Cardiology, vol. 66, no. 22, pp. 2498-2505, 2015.

[18] J. H. Badhiwala, F. Nassiri, W. Alhazzani et al., "Endovascular thrombectomy for acute ischemic stroke ameta-analysis," Journal of the American Medical Association, vol. 314, no. 17, pp. 1832-1843, 2015.

[19] M. Goyal, B. K. Menon, W. H. van Zwam et al., "Endovascular thrombectomy after large-vessel ischaemic stroke: a metaanalysis of individual patient data from five randomised trials," The Lancet, vol. 387, no. 10029, pp. 1723-1731, 2016.

[20] C. K. Bush, D. Kurimella, L. J. S. Cross et al., "Endovascular treatment with stent-retriever devices for acute ischemic stroke: a meta-analysis of randomized controlled trials," PLoS ONE, vol. 11, no. 1, Article ID e0147287, 2016.

[21] B. C. V. Campbell, M. D. Hill, M. Rubiera et al., "Safety and efficacy of solitaire stent thrombectomy: individual patient data meta-analysis of randomized trials," Stroke, vol. 47, no. 3, pp. 798-806, 2016.

[22] M. Lee, K.-S. Hong, and J. L. Saver, "Efficacy of intra-arterial fibrinolysis for acute ischemic stroke: meta-analysis of randomized controlled trials," Stroke, vol. 41, no. 5, pp. 932-937, 2010.

[23] J. D. Fields, P. Khatri, G. M. Nesbit et al., "Meta-analysis of randomized intra-arterial thrombolytic trials for the treatment of acute stroke due to middle cerebral artery occlusion," Journal of NeuroInterventional Surgery, vol. 3, no. 2, pp. 151-155, 2011.

[24] K. M. Fargen, D. Neal, D. J. Fiorella, A. S. Turk, M. Froehler, and J. Mocco, "A meta-analysis of prospective randomized controlled trials evaluating endovascular therapies for acute ischemic stroke," Journal of NeuroInterventional Surgery, vol. 7, no. 2, pp. 84-89, 2015. 


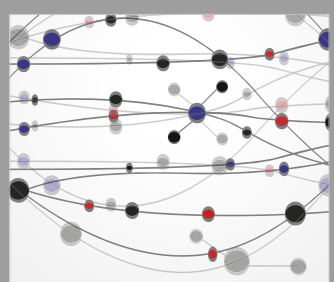

The Scientific World Journal
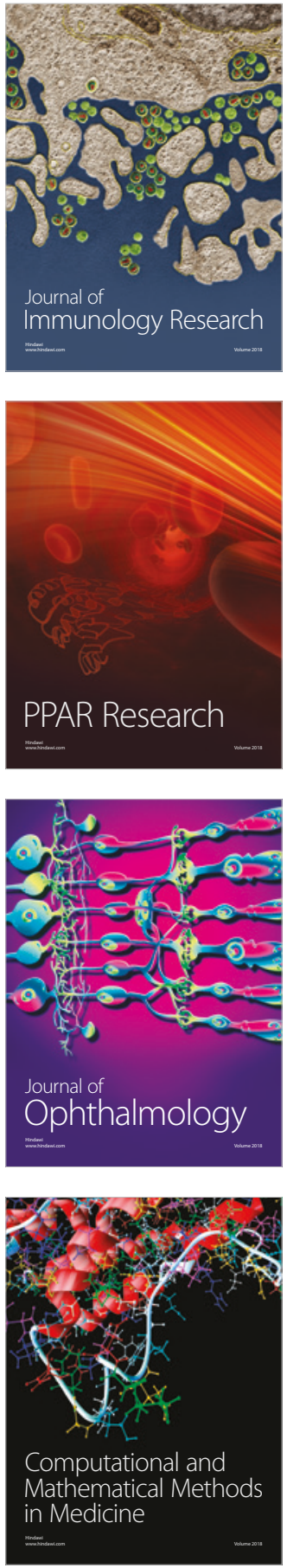

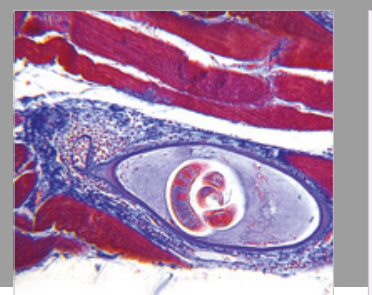

Gastroenterology Research and Practice

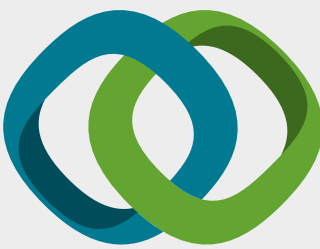

\section{Hindawi}

Submit your manuscripts at

www.hindawi.com
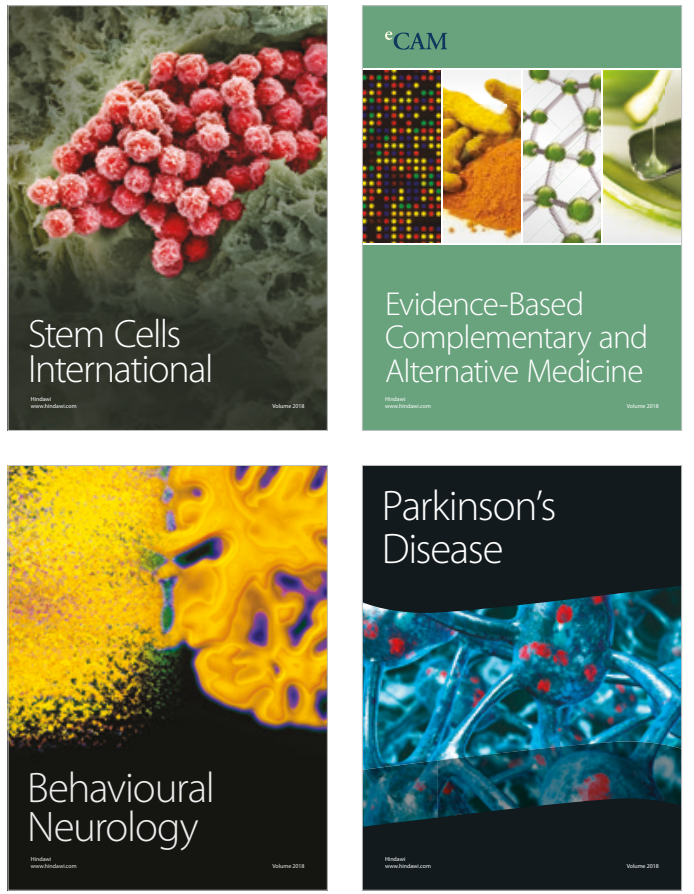

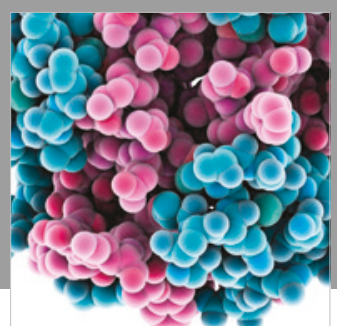

ournal of

Diabetes Research

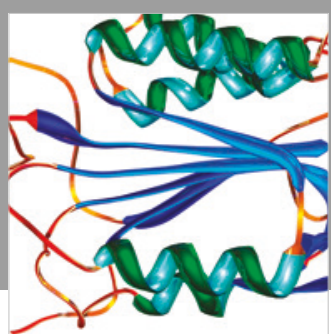

Disease Markers
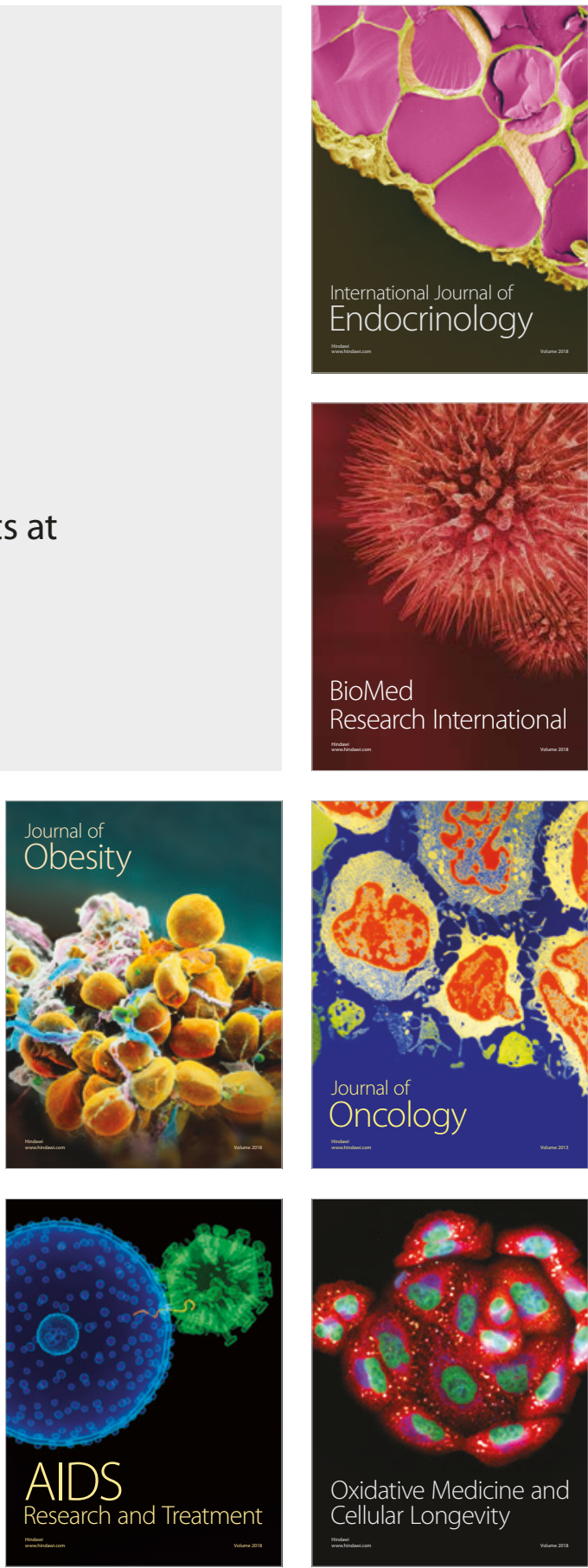